\title{
Does Non-Audit Service Compromise Audit Quality?
}

\author{
Hong-jo Park, Daegu University, South Korea \\ Jeong-un Choi, Daegu University, South Korea \\ Joonhei Cheung, Daegu University, South Korea
}

\begin{abstract}
This paper investigates the relationships between non-audit services, audit fee, audit hours and accounting quality. Previous studies have not provided consistent results for how simultaneous provision of audit and non-audit services by an independent auditor to a client company affects the audit quality. In addition, further studies have identified endogeneity in research method as the primary reason. Therefore, this study analyzed audit quality comprehensively using empirical analysis on data specific to Korea. This study employs research methods contrasting with existing studies in order to present a solution for the controversy related to the endogeneity from the effects of non-audit service provided by an independent auditor on audit quality. This study used audit compensation and abnormal accruals variables simultaneously, and audit time variable includes empirical data from Korean clients for comprehensive analysis. Study results found that the non-audit service significantly affects audit service quality before controlling for endogeneity. However, after controlling for endogeneity, even when the same independent auditor provides audit and non-audit services together, it did not affect the accounting quality.
\end{abstract}

Keywords: Non-Audit Services; Audit Time; Audit Fee; Audit Quality; Endogenous

\section{INTRODUCTION}

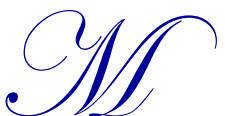

any studies have examined how audit quality is affected by an independent auditor providing audit and non-audit services together. However, the conclusions of these previous studies are not consistent, and various interpretations have been suggested for the relevance between audit service and non-audit service provision. Frankel, Johnson, and Nelson (2002) used abnormal accruals as an alternative variable of audit quality, and found that audit quality deteriorated as economic fee dependence is increased when both audit service and non-audit service are provided together. On the contrary, further study results have found no relevance from abnormal accruals when both audit and non-audit services are provided together (Ashbaugh, LaFond and Mayhew, 2003; Chung and Kallapur, 2003). Moreover, studies featuring audit compensation used as an alternative variable of audit quality found that if an independent auditor provides both audit and non-audit services, the audit service is increased, and therefore the impartiality of the independent auditor is not compromised even when non-audit service is simultaneously provided (Simunic, 1984; Palmrose, 1986).

Since there is no consensus concerning the relevance between audit service and non-audit service provided from the same auditor, many researchers have examined potential reasons, and have focused on the endogeneity of the research design as a possible factor. Whisenant, Sankaraguruswamy, and Raghunandan (2003) identified that the study result of Simunic (1984) and Palmrose (1986), which argues that audit compensation would be increased if both audit service and non-audit service are provided simultaneously, contained potential errors caused by the endogenous variable from regression analysis $(\mathrm{OLS})^{1}$. Moreover, as a result of multiple regression analysis using two-stage least squares (2SLS) in order to control the impact of such endogenous variables, it was verified that there is no relevance between audit service compensation and non-audit service compensation.

\footnotetext{
${ }^{1}$ Some subsequent studies disclosed that the results of existing studies are caused by endogeneity. For example, the study of Lawrence et al. (2011) found out that the study result of Becker et al. (1998) which states that the audit quality of Big 4 is relatively excellent was erroneous due to self-selection bias. They used matching model research method to control endogeneity, and as a result, Becker et al. (1998) verified that the audit quality is different because unique characteristics of companies under independent audit of Big 4 and non-Big 4, more specifically, the size of companies is fundamentally different rather than because the size of auditor is different.
} 
However, despite the argument of Whisenant et al. (2003), the relevance between non-audit service and audit service is still not clearly defined due to endogeneity and the alternative variables of audit quality. Therefore, this study expands the literature and comprehensively analyzes how the non-audit service provided from the same independent auditor affects the quality of audit service by applying empirical analysis and new research methods. Among the differentiated factors specified in this study, is that alternative variables measuring the quality of audit service are comprehensively examined. Existing research has analyzed the effects of non-audit service on the audit service quality, while either audit compensation or abnormal accruals was fragmentarily used as an alternative variable of audit quality. In comparison, this study uses audit compensation and abnormal accruals variables simultaneously, as well as audit time disclosed by clients in Korea.

The second differentiated factor in this study is the utilization of a more elaborate study method than previous studies in order to control endogenous variables. Most prior studies, including Whisenant et al. (2003), have used regression analysis by employing two-stage least squares (2SLS) for companies which received non-audit service, as well as companies which did not receive non-audit service, in order to control the effect of endogenous variables. Further studies have used regression analysis to compose fair-matched sample based on variables specific to companies. However, these methods cannot completely control the influence of self-selection bias on study samples. Therefore, this study conducted research on companies which received non-audit service in order to prevent discretionary factors in selecting samples and ensuring homogeneity of samples. Specifically, if a company received non-audit service at least once from the same independent auditor during the verification period, the effect of nonaudit service was analyzed by comparing audit service quality variables between years the non-audit service was provided and years with no non-audit service provided.

This study has important findings since it used variables and research methods which have not been used in previous studies. In particular, this study uses audit time data from Korea, and compared audit quality before and after nonaudit service is provided for companies which have received non-audit service in order to control endogeneity. Results of this study are meaningful since it can suggest important policies to regulate simultaneous provision of non-audit and audit services by the same independent auditor.

\section{LITERATURE REVIEW AND HYPOTHESES}

Previous studies have mainly focused on the independence of the auditor. Specifically, study results support the meaningful relationship between non-audit compensation and abnormal accruals (Frankel et al. 2002) and correlation between unspecified non-audit service and re-preparation of financial statements (Kinney, Palmrose, and Scholz 2004) suggest that the simultaneous provision of non-audit service may negatively affect the accounting quality for client companies. Specifically, the economic association between auditor and client companies from the simultaneous provision of non-audit service likely compromises the independence of the auditor, and this may negatively affect the accounting quality for client companies.

Conversely, Antle, Gordon, Narayanamoorthy, and Zhou (2006) found a significant negative relationship between non-audit compensation and abnormal accruals, and asserted that non-audit service may improve the accounting quality of client companies. This can be based on knowledge spillover due to simultaneous provision of audit and non-audit service (Simunic 1984).

However, DeFond, Raghunandan, and Subramanyam (2002) found an insignificant relationship between uncertain opinion on going concern and non-audit compensation (rate) and argued that the independence of auditor and nonaudit service is not relevant. In particular, a number of studies show that abnormal accruals are not relevant to nonaudit service (Chung and Kallapur 2003; Ashbaugh et al. 2003) and is contrary to the study of Frankel et al. (2002). In other words, while auditors can obtain additional benefits from non-audit service, they are also exposed to the loss of reputation due to compromised independence.

Previous studies on the simultaneous provision of non-audit service consist of three predominant viewpoints; audit quality is affected negatively, positively, or not related. In addition, the basis of these studies are compromised independence due to economic relationship (negative), knowledge spillover (positive) and loss of reputation (not related). This can be explained as audit quality composed of uncovering probability and reporting probability 
(DeAngelo 1981). That means, the effects of two factors will be likely found in combination as the compromised independence derives from decreased reporting probability and the knowledge spillover increases uncovering probability $^{2}$.

As audit (input) time and audit compensation are applied to the relationship between, the following assumptions can be expected: First of all, when audit and non-audit services are simultaneously provided, the audit time may be the same or less than when on-audit service is not provided. If simultaneous provision of non-audit service compromises the independence of auditor, the auditor will receive no benefit from uncovering accounting errors (wrongdoing) due to the relatively low report probability. Furthermore, since a more efficient audit is possible through knowledge spillover effects from non-audit service, it is unlikely that additional audit time is required. In addition, considering that audit compensation is determined mainly based on audit time provided, the audit compensation in the case where non-audit service is provided simultaneously may be the same or less compared to the case in which nonaudit service is not provided.

The estimation based on accruals (DA), audit time and audit compensation are mostly used as alternatives of audit quality. Specifically, when examining audit quality based on the simultaneous provision of non-audit service, the inconsistent results of previous studies may result from the endogeneity of audit and non-audit service rather than measurement error of audit time, audit compensation and abnormal accruals (Lawrence, Minutti-Meza, and Zhang $2011)^{3}$. Therefore, it is necessary to control the endogeneity in order to effectively examine the effects of the nonaudit service. Therefore, verification utilizes endogeneity control after null hypotheses are established as follows:

Hypothesis: There is no difference in audit quality between companies which receive non-audit service and companies which do not receive non-audit service.

Hypothesis 1-1: There is no difference in (provided) audit time between companies which receive non-audit service and companies which do not receive non-audit service.

Hypothesis 1-2: There is no difference in audit compensation between companies which receive non-audit service and companies which do not receive non-audit service.

Hypothesis 1-3: There is no difference in abnormal accruals between companies which receive non-audit service and companies which do not receive non-audit service.

\section{RESEARCH DESIGN}

\section{Research Model}

Methods for controlling endogeneity include the Heckman selection model (2SLS), the propensity score matching technique, the before and after comparison method, and the paired t-test. Lawrence et al. (2011) argued that studies should utilize the propensity score matching technique, which is an advanced version of the paried t-test, since the Heckman selection model cannot control endogeneity effectively. However, since the propensity score matching technique analyze endogeneity by finding similar paired sample, if there is no proper substitution sample, the result may be distorted and it will face variable selection issues. Moreover, although there are many differences in audit compensation and audit time according to industry characteristics, the propensity score matching technique does not match similar samples in the same industry. Therefore, the before and after comparison method is the most reasonable method to analyze the effect of non-audit service. Accordingly, this study compares audit compensation, audit time and audit quality by years for the companies which received non-audit service at least once according to the before and after comparison method.

\footnotetext{
${ }^{2}$ At this time, the reputation of auditor is 'acknowledged' audit quality, so if the loss of reputation is bigger than benefits from non-audit service, it likely increases the report probability.

${ }^{3}$ Simunic (1984) argues that the knowledge spillover effect can be through non-audit service. According to this, since more efficient audit is possible due to the knowledge spillover effect from non-audit service, we may expect less audit hour and less audit compensation. However, empirical results show that more audit compensation is given when non-audit service is provided.
} 
The research model the 1-1 and 1-2 hypotheses of this study as follows:

$$
\begin{aligned}
& \ln A F_{i t}=\alpha_{0}+\alpha_{1} \operatorname{lnNASFEE}\left(N A S_{D}\right)_{i t}+\alpha_{2} \text { FIRST }_{i t}+\alpha_{3} \text { ROA }_{i t-1}+\alpha_{4} \text { LOSS }_{i t-1}+\alpha_{5} \text { SIZE }_{i t-1}+ \\
& \alpha_{6} L E V_{i t-1}+\alpha_{7} G R W_{i t-1}+\alpha_{8} E X P_{R A T I O}{ }_{i t-1}+\alpha_{9} L I Q_{i t-1}+\alpha_{10} I N V A R_{i t-1}+\alpha_{11} M_{i t-1}+ \\
& \alpha_{12} \text { OWNER }_{i t}+\alpha_{13} \text { FORGI }_{i t}+\alpha_{14} \text { BIG }_{i t}+\alpha_{15} \text { OPINO }_{i t-1}+I N D+Y D+e \\
& \left.\ln A T_{i t}=\beta_{0}+\beta_{1} \ln N A S F E E(N A S) D\right)_{i t}+\beta_{2} F_{R S S T}+\beta_{3} R_{i t} O A_{i t-1}+\beta_{4} L_{0 S S} S_{i t-1}+\beta_{5} S_{I Z E_{i t-1}}+ \\
& \beta_{6} L E V_{i t-1}+\beta_{7} G R W_{i t-1}+\beta_{8} E_{\text {XPRATIO }} \beta_{i t-1}+\beta_{9} L I Q_{i t-1}+\beta_{10} I N V A R_{i t-1}+\beta_{11} M F_{i t-1}+ \\
& \beta_{12} O W N E R_{i t}+\beta_{13} F_{O R G I}+\beta_{14} \text { BIF }_{i t}+\beta_{15} O P I N O_{i t-1}+I N D+Y D+e
\end{aligned}
$$

$\ln A F: \quad$ The natural logarithm of audit fee;

$\ln A T: \quad$ The natural logarithm of audit hour;

InNASFEE: The natural logarithm of non-audit service fee;

NAS_D: $\quad 1$ if non-audit service and 0 otherwise;

FIRST: $\quad 1$ if first audit and 0 otherwise;

ROA: $\quad$ Net Income divided by beginning total assets;

LOSS: $\quad 1$ if a firm reports loss and 0 otherwise;

SIZE: $\quad$ The natural logarithm of total assets;

LEV: $\quad$ Total liabilities divided by beginning total assets;

$G R W: \quad$ Sales divided by beginning sales;

EXPRATIO: International sales divided by sales;

LIQ: $\quad$ Current assets divided by current liabilities

INVAR: $\quad$ Receivables and inventory divided by total assets;

$M B: \quad$ Market value divided by book value;

OWNER: $\quad$ Ownership of largest shareholder and related parties;

FORGI: $\quad$ Foreign ownership;

BIG4: $\quad 1$ if auditor is a large auditor (called Big 4) and 0 otherwise;

OPINO: $\quad 1$ if audit opinion is clean and 0 otherwise;

IND: $\quad$ Industry dummy

YD: $\quad$ Year dummy

The variables of interest in this study include $\operatorname{lnNASFEE~(non-audit~service~fee)~and~} N A S \_D$ (non-audit service). If endogeneity is controlled, there is no significant relationship between $\ln A T$ (audit time), and $\ln A F$ (audit fee) and InNASFEE. Therefore, we predict $\alpha 1$ and $\beta 1$ to be not significant in support of Hypothesis 1-1 and Hypothesis 1-2. We include control variables (FIRST, ROA, LOSS, SIZE, LEV, GRW, EXPRATIO, LIQ, INVAR, MB, OWNER, FORGI, BIG4, OPINO) consistent with Frankel et al. (2002) and Ashbaugh et al. (2003).

We estimate the relationship between the audit quality and non-audit service using Equation (3):

$$
\begin{aligned}
& D A_{i t}=\gamma_{0}+\gamma_{1} \operatorname{lnNASFEE}\left(N A S_{D}\right)_{i t}+\gamma_{2} S I Z E_{i t}+\gamma_{3} L E V_{i t}+\gamma_{4} G R W_{i t}+\gamma_{5} M_{i t}+\gamma_{6} O W N E R_{i t}+ \\
& \gamma_{7} \text { FORGI }_{i t}+\gamma_{8} \text { BIG }_{i t}+\gamma_{9} \text { KOSPI }_{i t-1}+I N D+Y D+e
\end{aligned}
$$

$D A: \quad$ Discretionary accruals;

KOSPI: $\quad 1$ if firms are listed in KOSPI and 0 otherwise;

Following previous studies, we use the discretionary accruals (DA) as our proxy for audit quality (e.g., Dechow , Sloan, and Sweeny 1995). We use a cross-sectional version of the modified Jones model. Furthermore, we utilize a cross-sectional model of discretionary accruals in which each year we estimate the model for every industry by its Kis-code. We expect that there is no significant relationship between audit quality and non-audit service. Therefore, we predict $\gamma_{1}$ to be not significant in support of Hypothesis 1-3. We include control variables (SIZE, LEV, GRW, MB, OWNER, FORGI, BIG4, KOSPI) as in Lawrence et al. (2011). 


\section{Selection of Sample}

We analyze the listed companies on the KOSPI and KOSDAQ from 2003 to 2014. We select those companies which meet the following conditions.

(1) Companies listed in KOSPI from 2002 to 2012

(2) Non-financial companies

(3) Companies which close their books in December

(4) Companies with data available for the analysis of KIS-VALUE and TS-2000

(5) Companies which present audit time in hours.

(6) Companies which belong to more than 10 industries by medium classification level

(7) Companies without impaired capital, not under administration, and not delisted

The data for non-audit service, audit compensation, and audit time is collected by using TS-2000. Moreover, companies which represent audit time in days or months rather than hours were excluded. In addition, companies which belong to less than 10 industries were not included in order to find abnormal accruals. The initial sample size, not controlling for endogeneity, is 11,578 observations (sample 2).

This study used before and after comparison method to control for endogeneity. The number of samples selected to use the before and after comparison method was 2,167 (sample 1).

\section{EMPIRICAL RESULTS}

\section{Descriptive Statistics and Correlation Analysis}

Table 1, Panel A shows descriptive statistics for the variables included in research models for sample 1. The mean $N A S_{-} D$ is $53.45 \%$ and the mean of $\ln N A S F E E$ is 9.1053 . The mean of $\ln A F$ is 18.0467 and $\ln A T$ is 6.6936 . Table 1, Panel B provides descriptive statistics for the variables included in research models for sample 2. For sample 2, the mean NAS_D is $28.95 \%$ and the mean of InNASFEE is 4.8669 .

Table 2, Panel A shows the Pearson correlation among the variables used in the research model for sample 1. The variable $N A S_{-} D$ shows no significance with the variables $\ln A F, \ln A T$, and $D A$. However, Panel B displays that the variable $N A S_{-} D$ shows a significant negative correlation at $1 \%$ significance level with variables $\ln A F, \ln A T$, and $D A$.

\section{Results for the Study Hypotheses}

Table 3 provides the results of Equation (1) for testing hypothesis 1-1. The results reveal no significant relationships between non-audit services fee $(\ln N A S F)$, non-audit services $\left(N A S_{-} D\right)$, and audit fee $(\ln A F)$ for sample 1 (controlling for endogeneity). However, the non-audit services fee, non-audit services, and audit hour have a significant positive correlation in sample 2 (non-controlling for endogeneity).

Table 4 provides the results of Equation (2) for testing hypothesis 1-2. Consistent with hypothesis 1-2, the results reveal no significant relationship between non-audit services fee $(\ln N A S F)$, non-audit services $\left(N A S \_D\right)$, and audit fee $(\ln A T)$ for sample 1 (Controlling for endogeneity). However, the non-audit services fee, non-audit services, and audit hour have a significant positive correlation in sample 2 (non-controlling for endogeneity).

Table 5 reports the results of Equation (3) for testing hypothesis 1-3. We find that the coefficients on $\ln N A S F$ and $N A S_{-} D$ are not significant for the second and third columns, which shows Equation (3) using sample 1. However, we find a positive relationship between $D A, \ln N A S F$, and $N A S \_D$. We find evidence generally supporting hypothesis $1-3$. 
Table 1. Descriptive Statistics

\begin{tabular}{|c|c|c|c|c|c|c|c|}
\hline \multicolumn{8}{|c|}{ Panel A. $n=2157$} \\
\hline Variables & Mean & Std. & Min & $1 Q$ & Median & $3 Q$ & Max \\
\hline $\ln A F_{t}$ & 18.0467 & 0.6450 & 16.9066 & 17.5997 & 17.9426 & 18.3264 & 20.0693 \\
\hline $\ln A T_{t}$ & 6.6936 & 0.7153 & 4.9698 & 6.2146 & 6.5889 & 7.0901 & 8.8247 \\
\hline$D A_{t}$ & -0.0046 & 0.1066 & -0.3834 & -0.0522 & -0.0019 & 0.0440 & 0.3359 \\
\hline $\ln N A S F$ & 9.1053 & 8.5514 & 0.0000 & 0.0000 & 15.2018 & 17.2167 & 19.8070 \\
\hline$N A S \_D_{t}$ & 0.5345 & 0.4989 & 0.0000 & 0.0000 & 1.0000 & 1.0000 & 1.0000 \\
\hline$S I Z \overline{E_{t}}$ & 25.7073 & 1.4006 & 23.2414 & 24.7111 & 25.4392 & 26.4828 & 30.0319 \\
\hline$L E V_{t}$ & 0.4093 & 0.1955 & 0.0406 & 0.2552 & 0.4101 & 0.5577 & 0.8664 \\
\hline$G R W_{t}$ & 1.1220 & 0.4072 & 0.3078 & 0.9371 & 1.0670 & 1.2031 & 3.2955 \\
\hline$M B_{t}$ & 1.4036 & 1.3641 & 0.1683 & 0.6086 & 0.9779 & 1.6807 & 8.8459 \\
\hline$R O A_{t}$ & 0.0103 & 0.1200 & -0.5898 & -0.0013 & 0.0322 & 0.0683 & 0.2266 \\
\hline$R O A_{t-1}$ & 0.0155 & 0.1193 & -0.5504 & 0.0030 & 0.0362 & 0.0749 & 0.2225 \\
\hline $\operatorname{LOSS}_{t-1}$ & 0.2388 & 0.4264 & 0.0000 & 0.0000 & 0.0000 & 0.0000 & 1.0000 \\
\hline$S I Z E_{t-1}$ & 25.6087 & 1.4080 & 23.0097 & 24.6205 & 25.3443 & 26.4007 & 29.8651 \\
\hline$L E V_{t-1}$ & 0.4094 & 0.1971 & 0.0504 & 0.2555 & 0.4086 & 0.5530 & 0.8863 \\
\hline$G R W_{t-1}$ & 1.1337 & 0.3994 & 0.3200 & 0.9461 & 1.0772 & 1.2278 & 3.0476 \\
\hline EXPRATIO ${ }_{t-1}$ & 0.2868 & 0.3159 & 0.0000 & 0.0001 & 0.1407 & 0.5485 & 0.9907 \\
\hline$L I Q_{t-1}$ & 2.6437 & 3.2509 & 0.2220 & 1.0627 & 1.6191 & 2.8020 & 23.8370 \\
\hline$I N V A R_{t-1}$ & 0.2802 & 0.1568 & 0.0000 & 0.1601 & 0.2667 & 0.3918 & 0.6696 \\
\hline$M V_{t-1}$ & 1.3452 & 1.2954 & 0.1356 & 0.5641 & 0.9324 & 1.6537 & 8.3174 \\
\hline$O W N E R_{t-1}$ & 0.2671 & 0.1366 & 0.0498 & 0.1629 & 0.2388 & 0.3483 & 0.6617 \\
\hline$F O R G I_{t-1}$ & 0.0690 & 0.1165 & 0.0000 & 0.0016 & 0.0141 & 0.0837 & 0.5535 \\
\hline$F I R S T_{t-1}$ & 0.1669 & 0.3730 & 0.0000 & 0.0000 & 0.0000 & 0.0000 & 1.0000 \\
\hline$B I G 4_{t-1}$ & 0.6078 & 0.4884 & 0.0000 & 0.0000 & 1.0000 & 1.0000 & 1.0000 \\
\hline OPINO $_{t-1}$ & 0.9977 & 0.0481 & 0.0000 & 1.0000 & 1.0000 & 1.0000 & 1.0000 \\
\hline $\mathrm{KOSPI}_{t}$ & 0.4483 & 0.4974 & 0.0000 & 0.0000 & 0.0000 & 1.0000 & 1.0000 \\
\hline \multicolumn{8}{|c|}{ Panel B. $n=11578$} \\
\hline Variables & Mean & Std. & Min & $1 Q$ & Median & $3 Q$ & Max \\
\hline $\ln A F_{t}$ & 18.0058 & 0.6417 & 16.8112 & 17.5958 & 17.9099 & 18.2582 & 20.2124 \\
\hline $\ln A T_{t}$ & 6.6663 & 0.7213 & 4.7185 & 6.2146 & 6.5765 & 7.0049 & 8.9872 \\
\hline$D A_{t}$ & -0.0036 & 0.0998 & -0.3520 & -0.0502 & -0.0013 & 0.0449 & 0.3043 \\
\hline $\ln N A S F$ & 4.8669 & 7.6628 & 0.0000 & 0.0000 & 0.0000 & 15.4249 & 19.9054 \\
\hline$N A S \_D_{t}$ & 0.2895 & 0.4536 & 0.0000 & 0.0000 & 0.0000 & 1.0000 & 1.0000 \\
\hline$S I Z E_{t}$ & 25.6612 & 1.3582 & 23.2805 & 24.7398 & 25.3954 & 26.3203 & 30.2485 \\
\hline$L E V_{t}$ & 0.4086 & 0.1963 & 0.0404 & 0.2518 & 0.4081 & 0.5553 & 0.8779 \\
\hline$G R W_{t}$ & 1.1049 & 0.3572 & 0.3342 & 0.9380 & 1.0590 & 1.1968 & 2.9226 \\
\hline$M B_{t}$ & 1.3333 & 1.2299 & 0.1848 & 0.5976 & 0.9500 & 1.5937 & 7.7098 \\
\hline$R O A_{t}$ & 0.0122 & 0.1140 & -0.5681 & -0.0007 & 0.0308 & 0.0678 & 0.2128 \\
\hline$R O A_{t-1}$ & 0.0177 & 0.1133 & -0.5504 & 0.0030 & 0.0342 & 0.0739 & 0.2235 \\
\hline $\operatorname{LOSS}_{t-1}$ & 0.2345 & 0.4237 & 0.0000 & 0.0000 & 0.0000 & 0.0000 & 1.0000 \\
\hline$S I Z E_{t-1}$ & 25.5854 & 1.3616 & 23.2381 & 24.6572 & 25.3218 & 26.2473 & 30.1817 \\
\hline$L E V_{t-1}$ & 0.4071 & 0.1952 & 0.0433 & 0.2517 & 0.4057 & 0.5520 & 0.8751 \\
\hline$G R W_{t-1}$ & 1.1296 & 0.4001 & 0.3405 & 0.9483 & 1.0704 & 1.2147 & 3.3131 \\
\hline$E_{X P R A T I O}$ & 0.2699 & 0.3054 & 0.0000 & 0.0001 & 0.1285 & 0.5005 & 0.9865 \\
\hline$L I Q_{t-1}$ & 2.5703 & 2.9318 & 0.2541 & 1.0514 & 1.5931 & 2.7697 & 19.2912 \\
\hline$I N V A R_{t-1}$ & 0.2782 & 0.1541 & 0.0000 & 0.1633 & 0.2670 & 0.3811 & 0.6785 \\
\hline$M V_{t-1}$ & 1.3051 & 1.1964 & 0.1757 & 0.5806 & 0.9343 & 1.5808 & 7.4121 \\
\hline$O W N E R_{t-1}$ & 0.2693 & 0.1382 & 0.0474 & 0.1655 & 0.2422 & 0.3482 & 0.6999 \\
\hline$F O R G I_{t-I}$ & 0.0633 & 0.1123 & 0.0000 & 0.0014 & 0.0114 & 0.0692 & 0.5613 \\
\hline$F I R S T_{t-1}$ & 0.1540 & 0.3610 & 0.0000 & 0.0000 & 0.0000 & 0.0000 & 1.0000 \\
\hline$B I G 4_{t-1}$ & 0.5449 & 0.4980 & 0.0000 & 0.0000 & 1.0000 & 1.0000 & 1.0000 \\
\hline OPINO $_{t-1}$ & 0.9977 & 0.0482 & 0.0000 & 1.0000 & 1.0000 & 1.0000 & 1.0000 \\
\hline $\mathrm{KOSPI}_{t}$ & 0.4222 & 0.4939 & 0.0000 & 0.0000 & 0.0000 & 1.0000 & 1.0000 \\
\hline
\end{tabular}


Table 2. Correlation Matrix

$\underline{\text { Panel A. Correlation Matrix for Sample } 1(n=2,157)}$

\begin{tabular}{|c|c|c|c|c|c|c|c|c|c|c|c|c|}
\hline & V1 & $\mathbf{V} 2$ & V3 & V4 & V5 & V6 & V7 & V8 & V9 & V10 & V11 & V12 \\
\hline $\ln A F_{t}(V 1)$ & 1.00 & & & & & & & & & & & \\
\hline $\ln A T_{t}(V 2)$ & 0.82 & 1.00 & & & & & & & & & & \\
\hline$D A_{t}(V 3)$ & -0.02 & -0.01 & 1.00 & & & & & & & & & \\
\hline $\ln N A S F_{t}(V 4)$ & 0.06 & 0.06 & 0.00 & 1.00 & & & & & & & & \\
\hline$N A S \_D_{t}(V 5)$ & 0.02 & 0.03 & 0.00 & 0.99 & 1.00 & & & & & & & \\
\hline$S I Z E_{t}(V 6)$ & 0.81 & 0.74 & 0.03 & 0.05 & 0.02 & 1.00 & & & & & & \\
\hline$L E V_{t}(V 7)$ & 0.29 & 0.21 & -0.05 & 0.01 & 0.00 & 0.26 & 1.00 & & & & & \\
\hline$G R W_{t}(V 8)$ & -0.04 & -0.06 & 0.09 & -0.01 & -0.01 & -0.02 & 0.07 & 1.00 & & & & \\
\hline$M V_{t}(V 9)$ & -0.01 & -0.03 & -0.04 & 0.02 & 0.02 & -0.16 & 0.09 & 0.10 & 1.00 & & & \\
\hline$R O A_{t}(V 10)$ & 0.07 & 0.08 & 0.41 & 0.01 & 0.01 & 0.23 & -0.23 & 0.15 & -0.12 & 1.00 & & \\
\hline$R O A_{t-1}(V 11)$ & 0.07 & 0.08 & 0.04 & -0.02 & -0.02 & 0.22 & -0.16 & -0.04 & -0.13 & 0.51 & 1.00 & \\
\hline $\operatorname{LOSS}_{t-1}(V 12)$ & -0.11 & -0.11 & -0.08 & -0.03 & -0.02 & -0.23 & 0.15 & 0.04 & 0.13 & -0.45 & -0.72 & 1.00 \\
\hline$S I Z E_{t-1}(V 13)$ & 0.82 & 0.75 & 0.00 & 0.05 & 0.02 & 0.98 & 0.26 & -0.09 & -0.18 & 0.19 & 0.20 & -0.21 \\
\hline$L E V_{t-1}(V 14)$ & 0.30 & 0.21 & -0.01 & 0.01 & 0.00 & 0.26 & 0.85 & 0.02 & 0.07 & -0.14 & -0.26 & 0.21 \\
\hline$G R W_{t-1}(V 15)$ & -0.01 & -0.04 & -0.07 & 0.00 & -0.01 & 0.01 & 0.05 & 0.01 & 0.10 & 0.08 & 0.19 & -0.14 \\
\hline$E_{\text {EXPATIO }}(V 16)$ & 0.01 & 0.02 & -0.02 & -0.01 & -0.01 & 0.03 & 0.05 & -0.04 & -0.01 & 0.03 & 0.04 & 0.01 \\
\hline$L I Q_{t-1}(V 17)$ & -0.21 & -0.15 & -0.02 & -0.01 & 0.00 & -0.21 & -0.50 & -0.01 & -0.01 & 0.02 & 0.10 & -0.07 \\
\hline$I N V A R_{t-1}(V 18)$ & -0.16 & -0.16 & -0.02 & -0.03 & -0.02 & -0.16 & 0.15 & -0.03 & -0.04 & $\mathbf{0 . 0 7}$ & 0.07 & -0.10 \\
\hline$M V_{t-1}(V 19)$ & 0.01 & -0.02 & 0.06 & 0.02 & 0.02 & -0.11 & 0.04 & 0.18 & 0.60 & -0.05 & -0.07 & 0.07 \\
\hline$O W N E R_{t-1}(V 20)$ & -0.02 & 0.01 & 0.03 & 0.01 & 0.01 & 0.04 & -0.08 & -0.04 & -0.10 & 0.15 & 0.14 & -0.12 \\
\hline FORGI $I_{t-1}(V 21)$ & 0.36 & 0.33 & 0.04 & 0.02 & 0.00 & 0.39 & -0.06 & -0.01 & 0.08 & 0.18 & 0.17 & -0.16 \\
\hline $\operatorname{FIRST}_{t-1}(V 22)$ & -0.03 & 0.00 & 0.01 & -0.04 & -0.03 & 0.01 & 0.00 & 0.07 & 0.04 & 0.02 & -0.04 & 0.05 \\
\hline$B I G 4_{t-1}(V 23)$ & 0.39 & 0.46 & 0.01 & 0.04 & 0.01 & 0.37 & 0.05 & -0.05 & -0.02 & 0.10 & 0.10 & -0.13 \\
\hline OPINO $_{t-1}(V 24)$ & -0.05 & -0.03 & -0.05 & -0.03 & -0.03 & -0.01 & -0.02 & 0.04 & -0.10 & -0.01 & 0.00 & -0.02 \\
\hline$K_{O S P I}(V 25)$ & 0.43 & 0.40 & 0.01 & 0.01 & -0.01 & 0.54 & 0.12 & -0.07 & -0.19 & 0.04 & 0.03 & -0.08 \\
\hline
\end{tabular}

Panel A continued

\begin{tabular}{|c|c|c|c|c|c|c|c|c|c|c|c|c|c|}
\hline & V13 & V14 & V15 & V16 & V17 & V18 & V19 & V20 & V21 & V22 & V23 & V24 & V25 \\
\hline$S I Z E_{t-1}(V 13)$ & 1.00 & & & & & & & & & & & & \\
\hline$L E V_{t-1}(V 14)$ & 0.26 & 1.00 & & & & & & & & & & & \\
\hline$G R W_{t-1}(V 15)$ & -0.01 & 0.07 & 1.00 & & & & & & & & & & \\
\hline EXPRATIO $_{t-1}(V 16)$ & 0.03 & 0.04 & 0.01 & 1.00 & & & & & & & & & \\
\hline$L I Q_{t-1}(V 17)$ & -0.21 & -0.58 & -0.07 & -0.04 & 1.00 & & & & & & & & \\
\hline$I N V A R_{t-1}(V 18)$ & -0.17 & 0.17 & 0.10 & 0.02 & -0.16 & 1.00 & & & & & & & \\
\hline$M V_{t-1}(V 19)$ & -0.15 & 0.10 & 0.13 & 0.01 & -0.01 & -0.04 & 1.00 & & & & & & \\
\hline$O W N E R_{t-1}(V 20)$ & 0.04 & -0.08 & -0.03 & -0.07 & 0.10 & 0.00 & -0.09 & 1.00 & & & & & \\
\hline FORGI $I_{t-1}(V 21)$ & 0.38 & -0.06 & -0.01 & 0.00 & 0.04 & -0.12 & $\mathbf{0 . 0 7}$ & 0.07 & 1.00 & & & & \\
\hline$F_{I R S T} T_{t-1}(V 22)$ & -0.01 & 0.02 & 0.00 & 0.01 & -0.01 & -0.01 & 0.05 & -0.01 & 0.01 & 1.00 & & & \\
\hline$B I G 4_{t-1}(V 23)$ & $\mathbf{0 . 3 7}$ & 0.04 & 0.00 & 0.01 & -0.04 & -0.08 & $\mathbf{0 . 0 0}$ & 0.11 & 0.27 & 0.00 & 1.00 & & \\
\hline $\operatorname{OPINO}_{t-1}(V 24)$ & -0.01 & -0.02 & -0.03 & 0.00 & 0.01 & 0.01 & -0.01 & -0.03 & -0.07 & 0.02 & 0.00 & 1.00 & \\
\hline $\mathrm{KOSPI}_{t}(\mathrm{~V} 25)$ & 0.55 & 0.14 & -0.08 & -0.05 & -0.12 & -0.07 & -0.20 & 0.03 & 0.24 & 0.01 & 0.24 & -0.03 & 1.00 \\
\hline
\end{tabular}

(Table 2, Panel B continued on next page) 
(Table 2, Panel B continued)

Panel B. Correlation Matrix for Sample $2(n=11,578)$

\begin{tabular}{|c|c|c|c|c|c|c|c|c|c|c|c|c|}
\hline & V1 & $\mathbf{V 2}$ & V3 & V4 & V5 & V6 & V7 & V8 & V9 & V10 & V11 & V12 \\
\hline $\ln A F_{t}(V 1)$ & 1.00 & & & & & & & & & & & \\
\hline $\ln A T_{t}(V 2)$ & 0.80 & 1.00 & & & & & & & & & & \\
\hline$D A_{t}(V 3)$ & -0.05 & -0.04 & 1.00 & & & & & & & & & \\
\hline $\ln N A S F_{t}(V 4)$ & 0.26 & 0.25 & -0.02 & 1.00 & & & & & & & & \\
\hline$N A S \_D_{t}(V 5)$ & 0.22 & 0.22 & -0.02 & 1.00 & 1.00 & & & & & & & \\
\hline$S I Z E_{t}(V 6)$ & 0.81 & 0.74 & 0.00 & 0.23 & 0.19 & 1.00 & & & & & & \\
\hline$L E V_{t}(V 7)$ & 0.22 & 0.16 & -0.07 & 0.00 & -0.01 & 0.20 & 1.00 & & & & & \\
\hline$G R W_{t}(V 8)$ & -0.04 & -0.05 & 0.10 & 0.01 & 0.01 & -0.02 & 0.05 & 1.00 & & & & \\
\hline$M V_{t}(V 9)$ & 0.06 & 0.03 & -0.05 & 0.05 & 0.04 & -0.10 & 0.07 & 0.10 & 1.00 & & & \\
\hline$R O A_{t}(V 10)$ & 0.05 & 0.06 & 0.40 & 0.06 & 0.06 & 0.20 & -0.28 & 0.19 & -0.10 & 1.00 & & \\
\hline$R O A_{t-1}(V 11)$ & 0.06 & 0.07 & 0.02 & 0.06 & 0.05 & 0.20 & -0.24 & -0.05 & -0.10 & 0.50 & 1.00 & \\
\hline $\operatorname{LOSS}_{t-1}(V 12)$ & -0.06 & -0.05 & -0.05 & -0.05 & -0.04 & -0.18 & 0.20 & 0.04 & 0.11 & -0.42 & -0.71 & 1.00 \\
\hline$S I Z E_{t-1}(V 13)$ & 0.81 & 0.74 & -0.03 & 0.22 & 0.19 & 0.99 & 0.20 & -0.08 & -0.11 & 0.14 & 0.17 & -0.16 \\
\hline$L E V_{t-1}(V 14)$ & 0.24 & 0.18 & -0.01 & 0.00 & 0.00 & 0.21 & 0.88 & 0.01 & 0.06 & -0.16 & -0.29 & 0.22 \\
\hline$G R W_{t-1}(V 15)$ & -0.02 & -0.03 & -0.02 & 0.01 & 0.01 & 0.00 & 0.02 & 0.01 & 0.08 & 0.10 & 0.19 & -0.16 \\
\hline EXPRATIO $_{t-1}(V 16)$ & 0.07 & 0.07 & -0.02 & 0.03 & 0.02 & 0.07 & 0.05 & -0.01 & 0.01 & 0.01 & 0.03 & 0.02 \\
\hline$L I Q_{t-1}(V 17)$ & -0.21 & -0.17 & 0.01 & 0.01 & 0.02 & -0.21 & -0.56 & -0.01 & 0.03 & 0.07 & 0.12 & -0.07 \\
\hline$I N V A R_{t-1}(V 18)$ & -0.17 & -0.17 & 0.00 & -0.08 & -0.07 & -0.16 & 0.17 & -0.03 & -0.07 & 0.09 & 0.09 & -0.09 \\
\hline$M V_{t-1}(V 19)$ & 0.07 & 0.04 & 0.01 & 0.05 & 0.04 & $\mathbf{- 0 . 0 7}$ & 0.02 & 0.11 & 0.67 & -0.04 & -0.07 & 0.07 \\
\hline$O W N E R_{t-1}(V 20)$ & 0.00 & 0.02 & 0.02 & 0.02 & 0.02 & 0.05 & -0.02 & -0.01 & -0.06 & 0.13 & 0.12 & -0.09 \\
\hline FORGI $I_{t-1}(V 21)$ & 0.44 & 0.38 & -0.01 & 0.19 & 0.17 & 0.46 & -0.10 & -0.01 & 0.11 & 0.18 & 0.18 & -0.15 \\
\hline $\operatorname{FIRST}_{t-1}(V 22)$ & -0.05 & -0.01 & 0.00 & -0.04 & -0.04 & -0.02 & 0.01 & 0.03 & 0.00 & -0.01 & -0.05 & 0.03 \\
\hline$B I G 4_{t-1}(V 23)$ & 0.38 & 0.43 & 0.00 & 0.19 & 0.17 & 0.36 & 0.03 & -0.02 & -0.01 & 0.09 & 0.10 & -0.09 \\
\hline OPINO $_{t-1}(V 24)$ & 0.00 & 0.01 & 0.00 & 0.01 & 0.01 & 0.02 & -0.04 & 0.03 & -0.01 & 0.04 & 0.03 & -0.03 \\
\hline $\mathrm{KOSPI}_{t}(V 25)$ & 0.43 & 0.40 & 0.01 & 0.14 & 0.12 & 0.55 & 0.10 & $\mathbf{- 0 . 0 7}$ & -0.17 & 0.08 & 0.06 & -0.09 \\
\hline
\end{tabular}

(Panel B continued)

\begin{tabular}{|c|c|c|c|c|c|c|c|c|c|c|c|c|c|}
\hline 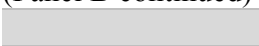 & V13 & V14 & V15 & V16 & V17 & V18 & V19 & V20 & V21 & V22 & V23 & V24 & V25 \\
\hline$S I Z E_{t-1}(V 13)$ & 1.00 & & & & & & & & & & & & \\
\hline$L E V_{t-1}(V 14)$ & 0.22 & 1.00 & & & & & & & & & & & \\
\hline$G R W_{t-1}(V 15)$ & -0.02 & 0.04 & 1.00 & & & & & & & & & & \\
\hline$E_{\text {XXRATIO }}(V 16)$ & 0.07 & 0.05 & 0.01 & 1.00 & & & & & & & & & \\
\hline$L I Q_{t-1}(V 17)$ & -0.22 & -0.62 & -0.03 & -0.01 & 1.00 & & & & & & & & \\
\hline$I_{N V A R_{t-1}}(V 18)$ & -0.17 & 0.19 & 0.08 & 0.03 & -0.14 & 1.00 & & & & & & & \\
\hline$M V_{t-1}(V 19)$ & -0.09 & 0.06 & 0.11 & 0.01 & 0.04 & -0.08 & 1.00 & & & & & & \\
\hline$O W N E R_{t-1}(V 20)$ & 0.04 & -0.02 & -0.01 & -0.06 & 0.04 & 0.02 & -0.06 & 1.00 & & & & & \\
\hline$F O R G I_{t-1}(V 21)$ & 0.46 & -0.09 & 0.01 & 0.01 & 0.03 & -0.13 & 0.11 & 0.02 & 1.00 & & & & \\
\hline $\operatorname{FIRST}_{t-1}(V 22)$ & -0.03 & 0.01 & -0.01 & -0.02 & 0.00 & -0.01 & 0.03 & 0.00 & -0.03 & 1.00 & & & \\
\hline$B I G 4_{t-1}(V 23)$ & 0.37 & 0.04 & -0.01 & 0.02 & -0.06 & -0.09 & 0.00 & 0.09 & 0.24 & -0.05 & 1.00 & & \\
\hline $\operatorname{OPINO}_{t-1}(V 24)$ & 0.01 & -0.02 & 0.00 & -0.01 & 0.01 & -0.01 & -0.01 & -0.01 & -0.01 & 0.01 & 0.02 & 1.00 & \\
\hline $\mathrm{KOSPI}_{t}(V 25)$ & 0.56 & 0.12 & -0.06 & -0.02 & -0.16 & -0.04 & -0.17 & 0.04 & 0.26 & 0.00 & 0.24 & 0.00 & 1.00 \\
\hline
\end{tabular}

1) The lower triangle presents the Pearson correlation coefficients. Boldfaced figures are statistically significant at the 0.05 level 
Table 3. Results of Hypothesis 1-1

\begin{tabular}{|c|c|c|c|c|}
\hline & \multicolumn{2}{|c|}{$\begin{array}{l}\text { Controlling for endogeneity } \\
\text { (Sample 1) }\end{array}$} & \multicolumn{2}{|c|}{$\begin{array}{c}\text { Non-controlling for endogeneity } \\
\text { (Sample 2) }\end{array}$} \\
\hline & $\begin{array}{c}\text { Coefficient } \\
\text { (t-stat.) }\end{array}$ & $\begin{array}{l}\text { Coefficient } \\
\text { (t-stat.) }\end{array}$ & $\begin{array}{l}\text { Coefficient } \\
\text { (t-stat.) }\end{array}$ & $\begin{array}{c}\text { Coefficient } \\
\text { (t-stat.) }\end{array}$ \\
\hline \multirow{2}{*}{ Intercept } & $9.3204^{* * *}$ & $9.3255^{* * *}$ & $8.9186^{* * *}$ & $8.8817^{* * *}$ \\
\hline & $(38.47)$ & $(38.45)$ & $(85.11)$ & $(84.78)$ \\
\hline \multirow{2}{*}{$\ln N A S F$} & 0.0007 & & $0.0050^{* * *}$ & \\
\hline & $(0.74)$ & & $(11.53)$ & \\
\hline \multirow{2}{*}{$N A S \_D$} & & 0.0024 & & $0.0718^{* * *}$ \\
\hline & & $(0.15)$ & & $(9.89)$ \\
\hline \multirow{2}{*}{ FIRST } & $-0.0667^{* * *}$ & $-0.0670^{* * *}$ & $-0.0531^{* * *}$ & $-0.0538^{* * *}$ \\
\hline & $(-3.32)$ & $(-3.33)$ & $(-5.89)$ & $(-5.96)$ \\
\hline \multirow{2}{*}{$R O A_{t-1}$} & $-0.3167^{* * *}$ & $-0.3199^{* * *}$ & $-0.3026^{* * *}$ & $-0.3039^{* * *}$ \\
\hline & $(-3.4)$ & $(-3.43)$ & $(-7.26)$ & $(-7.28)$ \\
\hline \multirow{2}{*}{$L O S S_{t-1}$} & -0.0026 & -0.0035 & $0.0210^{* *}$ & $0.0209^{* *}$ \\
\hline & $(-0.1)$ & $(-0.14)$ & $(1.98)$ & $(1.96)$ \\
\hline \multirow{2}{*}{$S I Z E_{t-1}$} & $0.3519^{* * *}$ & $0.3521^{* * *}$ & $0.3547^{* * *}$ & $0.3562^{* * *}$ \\
\hline & $(48.45)$ & $(48.49)$ & $(109.89)$ & $(110.54)$ \\
\hline \multirow{2}{*}{$L E V_{t-1}$} & $0.2445^{* * *}$ & $0.2443^{* * *}$ & $0.1563^{* * *}$ & $0.1556^{* *}$ \\
\hline & $(4.66)$ & $(4.65)$ & $(6.66)$ & $(6.62)$ \\
\hline \multirow{2}{*}{$G R W_{t-1}$} & -0.0275 & -0.0274 & $-0.0145^{*}$ & $-0.0144^{*}$ \\
\hline & $(-1.41)$ & $(-1.41)$ & $(-1.75)$ & $(-1.73)$ \\
\hline \multirow{2}{*}{$E_{X P R A T I O}$} & $-0.0499^{*}$ & $-0.0500^{*}$ & 0.0066 & 0.0063 \\
\hline & $(-1.89)$ & $(-1.90)$ & $(0.56)$ & $(0.53)$ \\
\hline \multirow{2}{*}{$L I Q_{t-1}$} & -0.00224 & -0.00224 & $-0.00496^{* * *}$ & $-0.00493^{* * *}$ \\
\hline & $(-0.8)$ & $(-0.80)$ & $(-3.55)$ & $(-3.53)$ \\
\hline \multirow{2}{*}{$I N V A R_{t-1}$} & -0.02053 & -0.02057 & 0.00674 & 0.0061 \\
\hline & $(-0.38)$ & $(-0.38)$ & $(0.29)$ & $(0.26)$ \\
\hline \multirow{2}{*}{$M B_{t-1}$} & $0.0463^{* * *}$ & $0.04643^{* * *}$ & $0.0545^{* *}$ & $0.055^{* * *}$ \\
\hline & $(7.27)$ & $(7.29)$ & $(18.68)$ & $(18.83)$ \\
\hline \multirow{2}{*}{$O W N E R_{t}$} & $-0.19042^{* * *}$ & $-0.19028^{* * *}$ & $-0.1315^{* * *}$ & $-0.13128^{* * *}$ \\
\hline & $(-3.39)$ & $(-3.39)$ & $(-5.65)$ & $(-5.63)$ \\
\hline \multirow{2}{*}{ FORGI $I_{t}$} & $0.24271^{* * *}$ & $0.24261^{* * *}$ & $0.36509^{* * *}$ & $0.36998^{* * *}$ \\
\hline & $(3.32)$ & $(3.32)$ & $(10.78)$ & $(10.92)$ \\
\hline \multirow{2}{*}{$B I G 4_{t}$} & $0.13175^{* * *}$ & $0.13214^{* * *}$ & $0.11199^{* * *}$ & $0.11332^{* * *}$ \\
\hline & $(7.87)$ & $(7.90)$ & $(16.06)$ & $(16.23)$ \\
\hline \multirow{2}{*}{ OPINO $_{t-1}$} & $-0.35837^{* *}$ & $-0.3606^{* *}$ & -0.09678 & -0.09635 \\
\hline & $(-2.32)$ & $(-2.34)$ & $(-1.48)$ & $(-1.47)$ \\
\hline IND & Included & Included & Included & Included \\
\hline YD & Included & Included & Included & Included \\
\hline Adj. $\mathrm{R}^{2}$ & 0.7227 & 0.7227 & 0.7217 & 0.7218 \\
\hline Obs. & 2,157 & 2,157 & 11,578 & 11,578 \\
\hline
\end{tabular}

1) Notes: ${ }^{*}, * * * *$ represent significance at the 10,5 , and 1 percent levels, respectively. 
Table 4. Results of Hypothesis 1-2

\begin{tabular}{|c|c|c|c|c|}
\hline & \multicolumn{2}{|c|}{$\begin{array}{c}\text { Controlling for endogeneity } \\
\text { (Sample 1) }\end{array}$} & \multicolumn{2}{|c|}{$\begin{array}{l}\text { Non-controlling for endogeneity } \\
\text { (Sample 2) }\end{array}$} \\
\hline & $\begin{array}{c}\text { Coefficient } \\
\text { (t-stat.) }\end{array}$ & $\begin{array}{l}\text { Coefficient } \\
\text { (t-stat.) }\end{array}$ & $\begin{array}{l}\text { Coefficient } \\
\text { (t-stat.) }\end{array}$ & $\begin{array}{l}\text { Coefficient } \\
\text { (t-stat.) }\end{array}$ \\
\hline \multirow{2}{*}{ Intercept } & $-1.7058^{* * *}$ & $-1.7068^{* * *}$ & $-2.1912^{* * *}$ & $-2.2347^{* * *}$ \\
\hline & $(-5.52)$ & $(-5.52)$ & $(-16.06)$ & $(-16.38)$ \\
\hline \multirow{2}{*}{$\ln N A S F$} & 0.0016 & & $0.0065^{* * *}$ & \\
\hline & $(1.41)$ & & $(11.55)$ & \\
\hline \multirow{2}{*}{$N A S \_D$} & & 0.0206 & & $0.0976^{* * *}$ \\
\hline & & $(1.04)$ & & $(10.34)$ \\
\hline \multirow{2}{*}{ FIRST } & 0.0041 & 0.0037 & $0.0437^{* * *}$ & $0.0430^{* * *}$ \\
\hline & $(0.16)$ & $(0.14)$ & $(3.72)$ & $(3.66)$ \\
\hline \multirow{2}{*}{$R O A_{t-1}$} & -0.1928 & $-0.1958^{*}$ & $-0.2066^{* * *}$ & $-0.2084^{* * *}$ \\
\hline & $(-1.62)$ & $(-1.65)$ & $(-3.81)$ & $(-3.84)$ \\
\hline \multirow{2}{*}{$\operatorname{LOSS}_{t-1}$} & 0.0389 & 0.0381 & $0.0532^{* * *}$ & $0.0530^{* * *}$ \\
\hline & $(1.20)$ & $(1.18)$ & $(3.84)$ & $(3.82)$ \\
\hline \multirow{2}{*}{$S I Z E_{t-1}$} & $0.3388^{* * *}$ & $0.3391^{* * *}$ & $0.3466^{* * *}$ & $0.3483^{* * *}$ \\
\hline & $(36.57)$ & $(36.62)$ & $(82.44)$ & $(83.03)$ \\
\hline \multirow{2}{*}{$L E V_{t-1}$} & 0.0513 & 0.0511 & -0.0094 & -0.0102 \\
\hline & $(0.77)$ & $(0.76)$ & $(-0.31)$ & $(-0.33)$ \\
\hline \multirow{2}{*}{$G R W_{t-1}$} & $-0.0452^{*}$ & $-0.0451^{*}$ & $-0.0218^{* *}$ & $-0.0217^{* *}$ \\
\hline & $(-1.82)$ & $(-1.82)$ & $(-2.02)$ & $(-2.01)$ \\
\hline \multirow{2}{*}{$E_{X P R A T I O}$} & 0.0062 & 0.0061 & $0.0417^{* * *}$ & $0.0415^{* * *}$ \\
\hline & $(0.18)$ & $(0.18)$ & $(2.72)$ & $(2.70)$ \\
\hline \multirow{2}{*}{$L I Q_{t-1}$} & 0.000372 & 0.000364 & $-0.00499^{* * *}$ & $-0.00498^{* * *}$ \\
\hline & $(0.10)$ & $(0.10)$ & $(-2.74)$ & $(-2.74)$ \\
\hline \multirow{2}{*}{$I N V A R_{t-1}$} & 0.04194 & 0.04184 & 0.00665 & 0.00598 \\
\hline & $(0.60)$ & $(0.60)$ & $(0.22)$ & $(0.19)$ \\
\hline \multirow{2}{*}{$M B_{t-1}$} & $0.02901^{* * *}$ & $0.02914^{* * *}$ & $0.04793^{* * *}$ & $0.0485^{* * *}$ \\
\hline & $(3.57)$ & $(3.59)$ & $(12.61)$ & $(12.75)$ \\
\hline \multirow{2}{*}{$O W N E R_{t}$} & $-0.19173^{* * *}$ & $-0.19169^{* * *}$ & $-0.0957^{* * *}$ & $-0.09559^{* * *}$ \\
\hline & $(-2.68)$ & $(-2.67)$ & $(-3.16)$ & $(-3.15)$ \\
\hline \multirow{2}{*}{$F O R G I_{t}$} & 0.1264 & 0.12662 & $0.16266^{* * *}$ & $0.16823^{* * *}$ \\
\hline & $(1.36)$ & $(1.36)$ & $(3.69)$ & $(3.81)$ \\
\hline \multirow{2}{*}{$B I G 4_{t}$} & $0.30784^{* * *}$ & 0.30848 & $0.2615^{* * *}$ & $0.26287^{* * *}$ \\
\hline & $(14.41)$ & $\left(14.45^{)^{* * *}}\right.$ & $(28.79)$ & $(28.92)$ \\
\hline \multirow{2}{*}{$O P I N O_{t-1}$} & -0.25469 & -0.25675 & -0.02889 & -0.02867 \\
\hline & $(-1.29)$ & $(-1.30)$ & $(-0.34)$ & $(-0.34)$ \\
\hline IND & Included & Included & Included & Included \\
\hline YD & Included & Included & Included & Included \\
\hline Adj. $\mathrm{R}^{2}$ & 0.6331 & 0.6330 & 0.6264 & 0.6255 \\
\hline Obs. & 2,157 & 2,157 & 11,578 & 11,578 \\
\hline
\end{tabular}

1) Notes: *,**,** represent significance at the 10,5 , and 1 percent levels, respectively. 
Table 5. Results of Hypothesis 1-3

\begin{tabular}{|c|c|c|c|c|}
\hline & \multicolumn{2}{|c|}{$\begin{array}{c}\text { Controlling for endogeneity } \\
\text { (Sample 1) }\end{array}$} & \multicolumn{2}{|c|}{$\begin{array}{c}\text { Non-controlling for endogeneity } \\
\text { (Sample 2) }\end{array}$} \\
\hline & $\begin{array}{c}\text { Coefficient } \\
\text { (t-stat.) }\end{array}$ & $\begin{array}{l}\text { Coefficient } \\
\text { (t-stat.) }\end{array}$ & $\begin{array}{l}\text { Coefficient } \\
\text { (t-stat.) }\end{array}$ & $\begin{array}{l}\text { Coefficient } \\
\text { (t-stat.) }\end{array}$ \\
\hline \multirow{2}{*}{ Intercept } & $0.2778^{* * *}$ & $0.2784^{* * *}$ & $0.1708^{* * *}$ & $0.1716^{* * *}$ \\
\hline & $(4.94)$ & $(4.95)$ & $(7.42)$ & $(7.47)$ \\
\hline \multirow{2}{*}{$\ln N A S F$} & -0.0001 & & $-0.0003^{* *}$ & \\
\hline & $(-0.48)$ & & $(-2.18)$ & \\
\hline \multirow{2}{*}{$N A S \_D_{t}$} & & -0.0021 & & $-0.0048^{* *}$ \\
\hline & & $(-0.48)$ & & $(-2.49)$ \\
\hline \multirow{2}{*}{$S I Z E_{t}$} & $-0.0113^{* * *}$ & $-0.0113^{* * *}$ & $-0.0070^{* * *}$ & $-0.0071^{* * *}$ \\
\hline & $(-5.05)$ & $(-5.06)$ & $(-7.61)$ & $(-7.66)$ \\
\hline \multirow{2}{*}{$L E V_{t}$} & $0.0491^{* * *}$ & $0.0491^{* * *}$ & $0.0362^{* * *}$ & $0.0361^{* * *}$ \\
\hline & $(3.90)$ & $(3.90)$ & $(7.28)$ & $(7.27)$ \\
\hline \multirow{2}{*}{$G R W_{t}$} & 0.0042 & 0.0042 & 0.0024 & 0.0024 \\
\hline & $(0.78)$ & $(0.78)$ & $(0.98)$ & $(0.98)$ \\
\hline \multirow{2}{*}{$M B_{t}$} & -0.0013 & -0.0013 & -0.0012 & -0.0012 \\
\hline & $(-0.76)$ & $(-0.76)$ & $(-1.64)$ & $(-1.64)$ \\
\hline \multirow{2}{*}{$R O A_{t}$} & $0.4221^{* * *}$ & $0.4221^{* * *}$ & $0.4017^{* * *}$ & $0.4018^{* * *}$ \\
\hline & $(21.10)$ & $(21.10)$ & $(47.62)$ & $(47.63)$ \\
\hline \multirow{2}{*}{$O W N E R_{t}$} & $-0.0304^{* * *}$ & $-0.0304^{*}$ & $-0.0250^{* * *}$ & $-0.0250^{* * *}$ \\
\hline & $(-1.90)$ & $(-1.90)$ & $(-4.03)$ & $(-4.02)$ \\
\hline \multirow{2}{*}{$F O R G I_{t}$} & 0.0060 & 0.0060 & $-0.0355^{* * *}$ & $-0.0355^{* * *}$ \\
\hline & $(0.29)$ & $(0.29)$ & $(-3.91)$ & $(-3.91)$ \\
\hline \multirow{2}{*}{$B I G 4_{t}$} & 0.0029 & 0.00284 & -0.00132 & -0.00128 \\
\hline & $(0.60)$ & $(0.60)$ & $(-0.71)$ & $(-0.69)$ \\
\hline \multirow{2}{*}{$\mathrm{KOSPI}_{t}$} & $0.0098^{*}$ & 0.00978 & 0.00611 & $0.00615^{* * *}$ \\
\hline & $(1.81)$ & $(1.81)$ & $(2.81)$ & $(2.83)$ \\
\hline IND & Included & Included & Included & Included \\
\hline YD & Included & Included & Included & Included \\
\hline Adj. $\mathrm{R}^{2}$ & 0.1796 & 0.1796 & 0.1797 & 0.1798 \\
\hline Obs. & 2,157 & 2,157 & 11,578 & 11,578 \\
\hline
\end{tabular}

1) Notes: ${ }^{*},{ }^{*}, * *$ represent significance at the 10,5 , and 1 percent levels, respectively.

\section{SUMMARY AND CONCLUSION}

Previous studies did not provide consistent results for how simultaneous provision of audit and non-audit services by an independent auditor to a client company affects audit quality. Researchers have identified endogeneity in the research method as the primary reason. Therefore, this study conducted analysis comprehensively using empirical data and research methods contrasting with existing studies in order to address the controversy related to endogeneity in effects of non-audit service by the same independent auditor with audit quality. This study used audit compensation and abnormal accruals variables simultaneously, and audit time variable using empirical data from Korean clients for comprehensive analysis. In addition, in order to control for endogeneity, the audit quality variable between before and after non-audit service is provided for companies which received non-audit service only.

Study results found that the non-audit service meaningfully affects audit service quality before controlling for endogeneity. However, after controlling for endogeneity, the same independent auditor providing non-audit service did not affect the audit service quality. In other words, it was found that the non-audit service did not affect the quality of external audit after endogeneity from self-selection bias is removed and the homogeneity of samples is ensured by comparing the audit quality before and after the non-audit service was provided for companies at least once from the same independent auditor. Furthermore, this result was consistent using all audit compensation variables of audit quality, abnormal accruals and audit time variable.

Result of this study are differentiated from previous studies as it selects companies which receive non-audit service and controls for endogeneity by comparing the audit quality variable before and after non-audit service is provided 
for the same company. Furthermore, this study uses audit time data specifically disclosed in Korea. This study has meaningful implications for policies which can regulate the simultaneous provision of non-audit and audit services using the same independent auditor.

\section{REFERENCES}

Ashbaugh, H., LaFond, R. \& Mayhew, B. (2003). Do non-audit services compromise auditor independence? Further evidence. The Accounting Review 78(3), 611-639.

Antle, R., Gordon, E., Narayanamoorthy, G. \& Zhou, L. (2006). The joint determination of audit fees, non-audit fees, and abnormal accruals. Review of Quantitative Finance and Accounting 27(3), 235-266.

Chung H. and Kallapur, S. (2003), Client importance nonaudit services and abnormal accruals, The Accounting Review 78(4), 931-955.

DeAngelo, L. E. (1981). Auditor size and audit quality, Journal of Accounting and Economics 3: 183-199.

Dechow, P., Sloan, R., \& Sweeny, A. (1995), Detecting earnings management, The Accounting Review 70 (2), 193-225.

DeFond, M. L., Raghunandan K., \& Subramanyam K. R. (2002). Do non-audit service fee impair auditor independence? Evidence from going concern audit opinions, Journal of Accounting Research 40(4): 1247-1274.

Frankel, R. M., Johnson, M. F., \& Nelson K. K. (2002). The relation between auditors' fee for nonaudit services and earnings management, The Accounting Review 77(Supplement): 71-105.

Kinney Jr., W. R., Palmrose, Z., \&Scholz, S. (2004). Auditor independence, non-audit services, and restatements: Was the U. S. government right? Journal of Accounting Research 42 (3): 561-588.

Lawrence A., Minutti-Meza, M., and Zhang, P. (2011). Can Big4 versus non-Big4 differences in audit quality proxies be attributed to client characteristics?, The Accounting Review 86(1), 259-286.

Palmrose, Z. (1986). Audit Fee and Auditor Size: Further Evidence, Journal of Accounting Research 24(1), 97-110.

Simunic, D. A. (1984). Auditing, consulting, and auditor independence, Journal of Accounting Research 22(2): 679-702.

Whisenant, S., Sankaraguruswamy, S. \& Raghunandan K. (2003). Evidence on the joint determination of Audit and non-audit fees. Journal of Accounting Research 41(4): 721-744. 